\title{
IMMUNOHISTOCHEMICAL AND IMMUNOELECTRON MICROSCOPICAL INVESTIGATION OF TAU-POSITIVE NEURONS LACKING TANGLE-FORMATION IN PROGRESSIVE SUPRANUCLEAR PALSY
}

\author{
Feng Li ${ }^{1}$, Eizo IseKI ${ }^{1,3}$, KenJI KosaKa ${ }^{1}$ and KenJI IKeDA ${ }^{2}$ \\ ${ }^{1}$ Department of Psychiatry, Yokohama City University School of Medicine, Fukuura, Kanazawaku, Yokohama, 236-0004, and \\ ${ }^{2}$ Tokyo Institute of Psychiatry, 2-1-8 Kamikitazawa, Setagayaku, Tokyo, 156-0057, Japan
}

\begin{abstract}
Tau-positive neurons lacking tangle-formation (TNLT) show diffuse or fine granular cytoplasmic staining with the tau-immunohistochemistry, and are more frequently found in progressive supranuclear palsy (PSP) and corticobasal degeneration (CBD) than in Alzheimer's disease (AD). In this study, the regional incidence and ultrastructure of TNLT in 9 PSP cases were examined. The regional proportion of TNLT in tau-positive neurons was especially high in the hippocampus and amygdala, although the incidence of TNLT was dependent on the case and region. Ultrastructurally, numerous tau-positive ribosomelike granules were found in the perikarya and dendrites of TNLT, but paired helical filaments (PHF) and straight tubules were not identified. These findings suggest that TNLT in PSP or CBD are not the early stage of neurofibrillary tangles (NFT) unlike TNLT in AD, but are another form of abnormally phosphorylated tau.
\end{abstract}

Tau-positive neurons lacking tangle-formation (TNLT) have been so far described under various nomenclatures such as pretangle neurons $(12,25)$, neurons lacking neurofibrillary tangles (NFT) (23), stage 0 tangles (2) and diffuse tau staining (22), and have been identified in Alzheimer's disease (AD) $(2,9-12,18,19,21,22,27)$, progressive supranuclear palsy (PSP) $(6,13,15,21,23)$, corticobasal degeneration (CBD) $(8,16,17,25,26)$, parkinsonism-dementia complex of Guam (11) and aged non-demented individuals $(2,14,22)$. TNLT show diffuse or fine granular cytoplasmic staining with the Gallyas-Braak silver stain or the tauimmunohistochemistry $(2,12,23)$, although they are morphologically normal-shaped neurons. Ultrastructurally, TNLT in AD have many ribosomes and diffusely scattered abnormal fibers within the neuronal perikarya. Some of these abnormal fibers are presented as paired helical filaments (PHF) and

${ }^{3}$ Correspondence to: E. Iseki at the above address. Tel: (81)45-787-2667; Fax: (81)45-783-2540 others as straight fibers with granular materials (2, 19, 23). Bancher et al. (2) suggested that accumulation of abnormally phosphorylated tau is one of the earliest cytoskeletal changes in the process of tangle-formation in $\mathrm{AD}$, and that TNLT represented an early stage of NFT formation. TNLT are more frequently found in CBD and PSP than in $\mathrm{AD}$, and are particularly characteristic of $\mathrm{CBD}(6$, 15-17, 25, 26). Nevertheless, it remains unclear whether TNLT are the early stage of NFT in CBD and PSP, because the ultrastructure of TNLT in CBD and PSP is largely unknown.

In this study, we investigated the regional incidence of TNLT with various anti-cytoskeletal protein antibodies and the ultrastructure of TNLT using tau-immunoelectron microscopy in PSP, and discussed the relationship between TNLT and NFT in PSP.

\section{MATERIALS AND METHODS}

In this study, 9 pathologically verified PSP cases 
Table 1 Semiquantitative Analysis of the Regional Incidence of TNLT

\begin{tabular}{|c|c|c|c|c|c|c|c|c|c|c|c|c|c|}
\hline Case & Age $(y) / \operatorname{Sex}$ & Duration $(y)$ & B.W. (g) & SFC & $\mathrm{PC}$ & $\mathrm{EC}$ & TEC & Hipp & Amy & Mey & Hypo & Pal & Sub \\
\hline 1 & $57 / \mathrm{M}$ & 6 & 1,280 & + & + & + & - & - & + & + & + & - & - \\
\hline 2 & $62 / F$ & 9 & 1,015 & ++ & $\mathrm{NA}^{\prime}$ & ++ & ++ & +++ & ++ & + & ++ & + & + \\
\hline 3 & $66 / \mathrm{M}$ & 5 & 970 & - & + & + & + & - & + & + & + & + & $\mathrm{NA}$ \\
\hline $4^{\mathrm{a}}$ & $67 / \mathrm{M}$ & 3 & 1,160 & ++ & ++ & ++ & ++ & +++ & ++ & ++ & ++ & + & + \\
\hline 5 & $67 / \mathrm{M}$ & 8 & 1,350 & + & + & - & + & + & $+t$ & + & + & + & + \\
\hline 6 & $69 / \mathrm{M}$ & 4 & 1,280 & - & + & + & + & ++ & + & + & + & - & + \\
\hline 7 & $71 / \mathrm{M}$ & 4 & 1,250 & ++ & ++ & + & ++ & +++ & ++ & + & + & + & ++ \\
\hline 8 & $76 / \mathrm{M}$ & 10 & 1,200 & + & + & + & + & ++ & ++ & + & + & + & ++ \\
\hline 9 & $80 / \mathrm{F}$ & 5 & 1,150 & - & - & + & + & ++ & NA & + & + & + & + \\
\hline
\end{tabular}

TNLT, tau-positive neurons lacking tangle-formation; B.W., brain weight; SFC, superior frontal cortex; PC, precentral cortex; EC, entorhinal cortex; TEC, transentorhinal cortex; Hipp, hippocampus; Amy, amygdala; Mey, Meynert nucleus; Hypo, hypothalamus; Pal, pallidum; Sub, subthalamus. - , none; + , a few; ++ , moderate; +++ , frequent; NA, not available

aLi et al., 1996

(5) were examined (Table 1). The brains were fixed in $4 \%$ paraformaldehyde in $0.1 \mathrm{M}$ phosphate buffer, pH 7.4. The cerebral hemispheric slices were embedded in paraffin, cut into $8 \mu \mathrm{m}$-thick sections and stained with the hematoxylin-eosin, Klüver-Barrera, Holzer, methenamine-silver and Gallyas-Braak methods for pathological examination.

For the immunohistochemistry, 9 kinds of primary antibodies were used in this study (Table 2). Alz-50 and Human-tau, TF-11, and Ab71 are the antibodies for the amino half of tau, the middle region of tau, and the carboxy-terminal region of tau, respectively. Ab39 is the antibody for ADNFT. SMI 31 and SMI 32 are the antibodies for phosphorylated and non-phosphorylated neurofilaments, respectively. MAB1510 and MAP2 are the antibodies for ubiquitin and microtubule-associated protein 2, respectively. These primary antibodies were detected using the avidin-biotinylated HRP complex (ABC) method (Elite Kit, Vector, U.S.A.) and visualized with DAB (Sigma, U.S.A.).

For the tau-immunoelectron microscopy, the tissue sample including the CA2-3 of the hippocampus from case 4 was cut into $40 \mu \mathrm{m}$-thick vibratome sections and immunostained with $\mathrm{Alz}$ 50 (dilution, 1:50) by the $\mathrm{ABC}$ procedure. The sections were post-fixed with $2.5 \%$ glutaraldehyde and $2 \% \mathrm{OsO}_{4}$, embedded in epon, dissected in ultrathin sections and stained with lead citrate.

\section{RESULTS}

The immunohistochemical staining properties of tau-positive neurons in the examined cases are summarized in Table 3. Tau-positive neurons consisted of NFT and TNLT. NFT demonstrated tangle-formation, but TNLT showed normal morphological appearance and diffusely granular cytoplasmic staining without tangle-formation (Fig, 1A). Alz-50 and Human-tau immunostained both NFT and TNLT, while Ab71 and Ab39 immunostained only a part of NFT. MAB1510 immunostained a part of NFT and a small number of TNLT.

Semiquantitative regional incidence of TNLT immunostained by Human-tau is shown in Table 1 . The incidence of TNLT was dependent on the case and region. The regional proportion of TNLT in tau-positive neurons was especially high in the hippocampus and amygdala.

In the tau-immunoelectron microscopic findings of TNLT in the hippocampal CA2-3 (Fig. 1B), numerous tau-positive ribosome-like granules were

Fig. 1 A: NFT (arrow) and TNLT (arrowhead) in the amygdala (Human-tau immunostaining). B: TNLT and neuropil threads in the hippocampal CA2-3 (Alz-50 immunostaining)

Fig. 2 A: Immunoelectron microscopic appearance of a TNLT. $\times 2,200$. B: A higher magnification; numerous tau-positive ribosome-like granules are found in the perikaryon, mixed with the organelles. $\times 30,800$. C: Immunoelectron microscopic appearence of a neuropil thread, corresponding to the dendrite of TNLT. Arrow shows a presynaptic terminal. $\times 8,500$. D: A higher magnification; Many tau-positive ribosome-like granules and some tau-negative neurofilaments of $10-15 \mathrm{~nm}$ in diameter (arrows) are found. $\times 33,700$ 


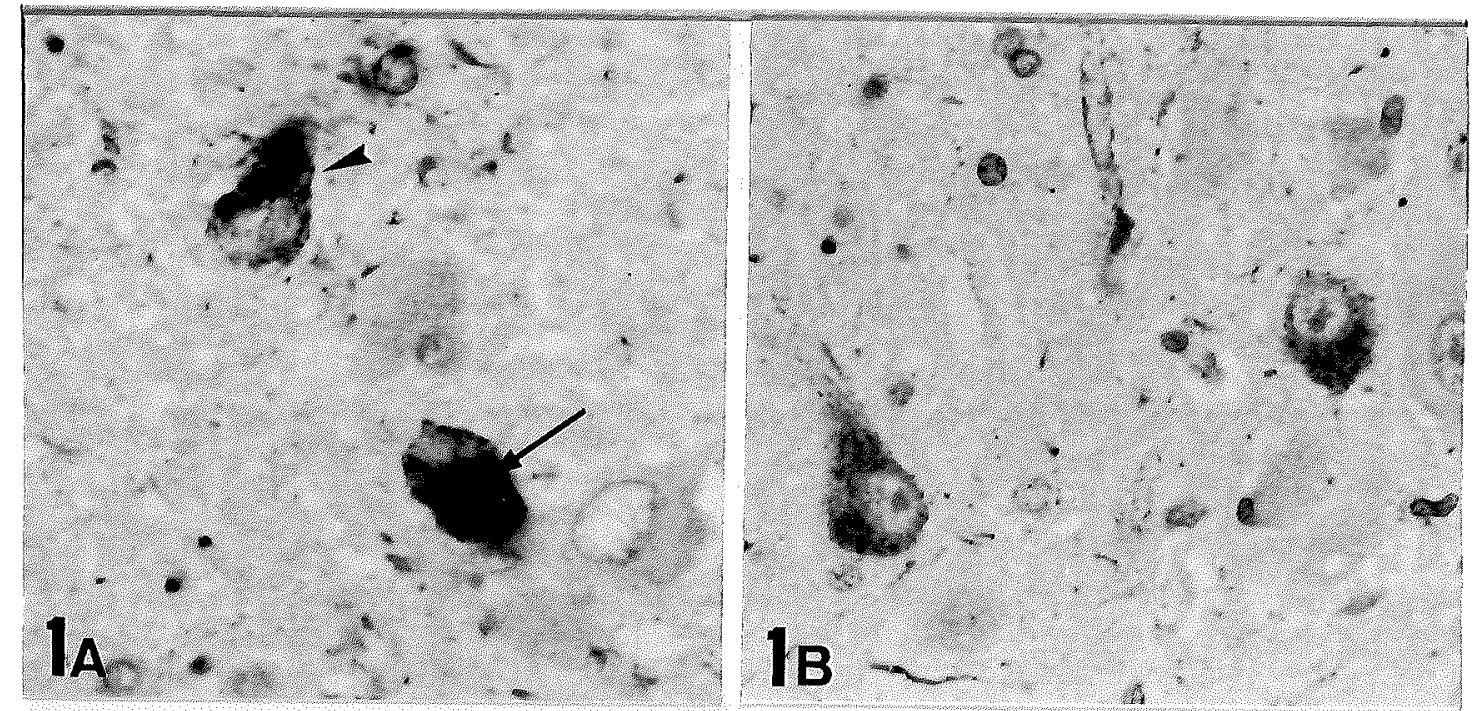

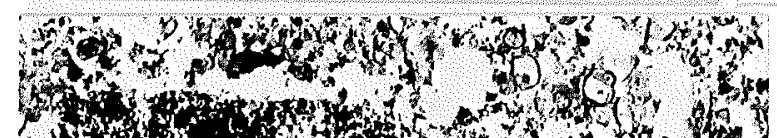

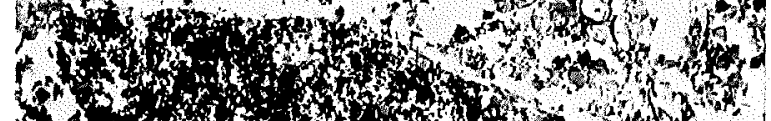
5,

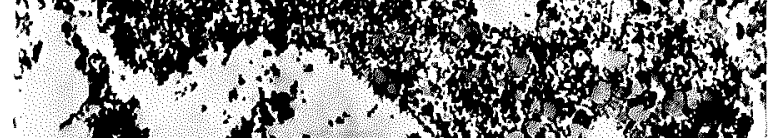
(

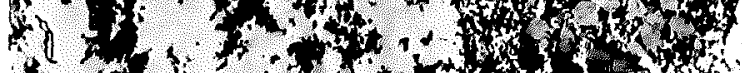

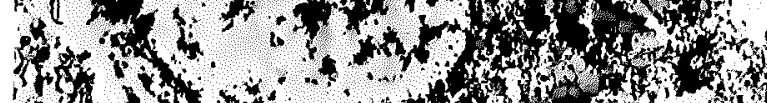
Ho

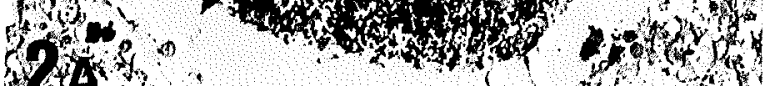

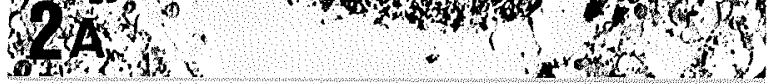

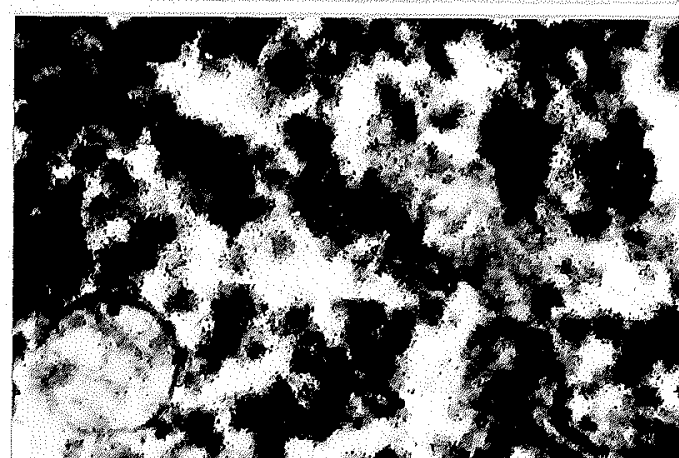

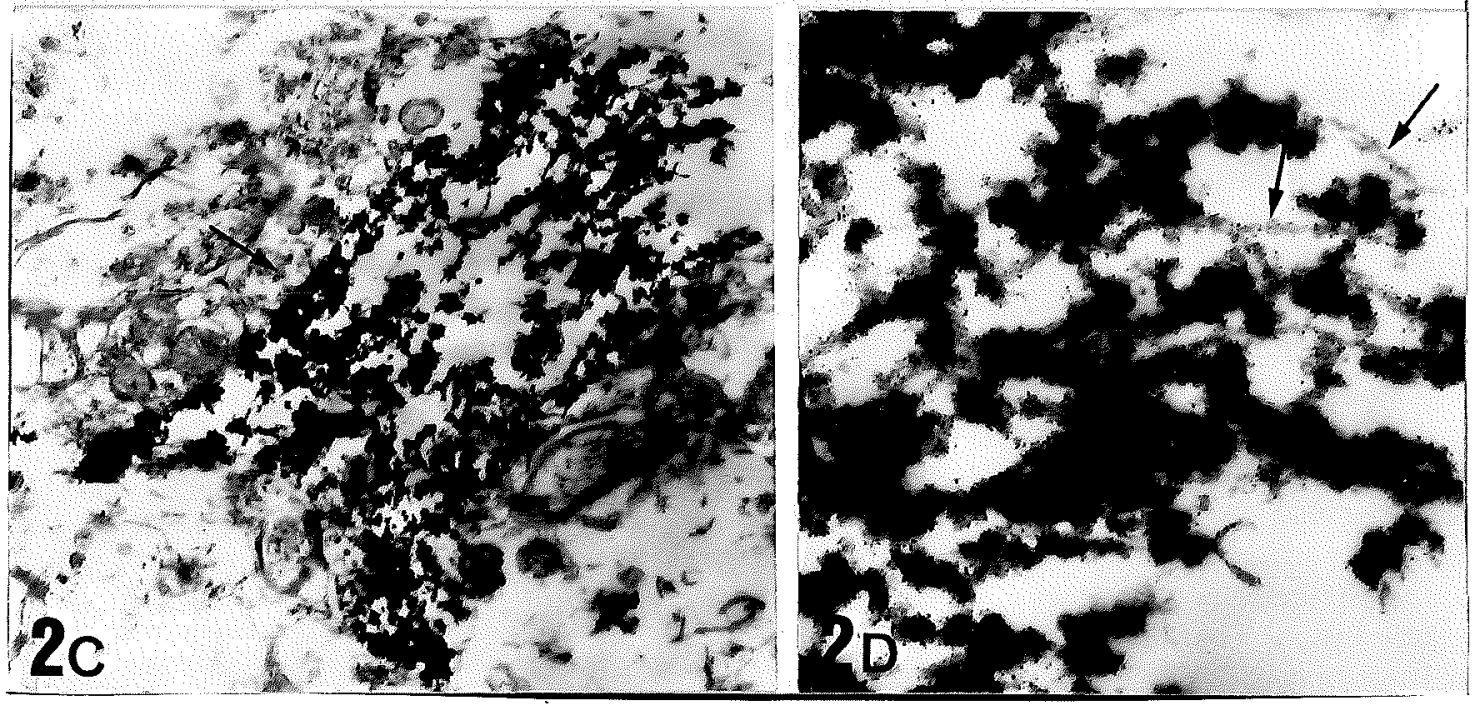

Figs. 1 and 2 
Table 2 Antibodies Used for Immunohistochemical Studies

\begin{tabular}{lllcll}
\hline Antibody & Species & \multicolumn{1}{c}{ Type } & Isotype & \multicolumn{1}{c}{ Source } & Dilution \\
\hline Alz-50 & Mouse & Monoclonal & $\operatorname{IgM}$ & Dr Davies & $1: 200$ \\
Human-tau & Rabbit & Polyclonal & & Dr Ihara & $1: 2,000$ \\
TF-11 & Mouse & Monoclonal & & Dr Mori & $1: 30$ \\
Ab71 & Mouse & Monoclonal & $\mathrm{IgG}_{1}$ & Dr Yen & $1: 2,000$ \\
Ab39 & Mouse & Monoclonal & $\mathrm{IgG}_{1}$ & Dr Yen & $1: 2,000$ \\
MAB1510 & Mouse & Monoclonal & $\mathrm{IgG}_{1}$ & Chemicon & $1: 8,000$ \\
SMI31 & Mouse & Monoclonal & $\mathrm{IgG}_{1}$ & Sternberger & $1: 16,000$ \\
SMI32 & Mouse & Monoclonal & $\mathrm{IgG}_{1}$ & Sternberger & $1: 8,000$ \\
MAP2 & Mouse & Monoclonal & $\mathrm{IgG}_{1}$ & Chemicon & $1: 1,000$ \\
\hline
\end{tabular}

Table 3 Immunohistochemical Staining Properties of Tau-Positive Neurons

\begin{tabular}{lcc}
\hline Antibody & NFT & TNLT \\
\hline Alz-50 & ++ & $+1++$ \\
Human-tau & ++ & + I++ \\
TF-11 & + & \pm \\
Ab71 & + & - \\
Ab39 & + & - \\
MAB1510 & + & \pm \\
SMI31 & \pm & - \\
SMI32 & - & - \\
MAP2 & \pm & - \\
\hline
\end{tabular}

NFT, neurofibrillary tangles; TNLT, tau-positive neurons lacking tangle-formation; -, negative; \pm , weakly positive; + , positive; ++ , strongly positive

found in the perikarya of TNLT, mixed with the organelles and lipofuscin granules. A few neurofilaments of $10-15 \mathrm{~nm}$ in diameter were tau-negative, although they were partly coated with tau-positive granules (Fig. 2, A and B). PHF and straight tubules were not identified. Many tau-positive ribosome-like granules and some tau-negative neurofilaments were also found in the neuropil threads around TNLT, corresponding to the dendrites of TNLT (Fig. 2, C and D).

\section{DISCUSSION}

In this study, TNLT were identified in the cerebral cortex and subcortical nuclei of all the examined cases, although their incidence was dependent on the case and region. The distribution of TNLT was different from that of NFT in PSP $(3,4,6,7)$ and their proportion in tau-positive neurons was especially high in the hippocampus and amygdala.
With the tau-immunoelectron microscopy, numerous tau-positive ribosome-like granules were found in the perikarya and dendrites of TNLT, but tau-positive fibrillary structures including PHF and straight tubules were not identified in TNLT. These ultrastructural appearances are different from those of PSP- or CBD-NFT $(1,20,24,26,28)$, suggesting that there is no transitional form between TNLT and NFT in PSP. The ultrastructure of TNLT in CBD is considered to be identical to that in PSP, due to the cytopathological similarity between PSP and $\mathrm{CBD}$.

In this study, it is suggested from the distribution, antigenicity and ultrastructure of TNLT that TNLT in PSP or CBD are not the early stage of NFT unlike TNLT in $\operatorname{AD}(2,19)$, but are another form of abnormally phosphorylated tau. In the future, the cytoskeletal abnormality should include the cytoskeletal protein abnormality without abnormal filaments.

This study was partly supported by a grant-in-aid for scientific research from the Ministry of Education, Science and Culture, Japan. We are grateful to Drs P. Davies, Y. Ihara, K. Mori and S.-H. Yen for their generous supply of antibodies.

Received 27 January 1998; and accepted 16 February 1998

\section{REFERENCES}

1. Arima K., Uesugi H., Fujtta I., Sakurai Y., Oyanagi S., ANDoH S., IzumiYama Y. and Inose T. (1994) Corticonigral degeneration with neuronal achromasia presenting with primary progressive aphasia: ultrastructural and immunocytochemical studies. J. Neurol. Sci. 127, 186-197

2. Bancher C., Brunner C., Lassmsnn H., Budka H., Jellinger K., Wiche G., Seitelberger F., GrundkeIQBAL K. and WISNIEWSKI H. M. (1989) Accumulation of abnormally phosphorylated tau precedes the formation of neurofibrillary tangles in Alzheimer's disease. Brain Res. 
$477,90-99$

3. BraAk H., Jellinger K., BraAk E. and Bohl J. (1992) Allocortical neurofibrillary changes in progressive supranuclear palsy. Acta Neuropathol. 84, 478-483

4. Hanihara T., Amano N., Takahashi T., Nagatomo H. and YAGISHITA S. (1995) Distribution of tangles and threads in the cerebral cortex in progressive supranuclear palsy. Neuropathol. Appl. Neurobiol. 21, 319-326

5. Hauw J. J., Daniel S. E., Dickson D., Horoupian D.S., Jellinger K., Lantos P. L., McKee A., Tabaton M. and LiTVAN I. (1994) Preliminary NINDS neuropathologic criteria for Steele Richardson-Olszewski syndrome (progressive supranuclear palsy). Neurology 44, 2015-2019

6. Hauw J. J., Verny M., Delaere P., Cervera P., He Y. and DuYCKAERTS C. (1990) Constant neurofibrillary changes in the neocortex in progressive supranuclear palsy. Basic difference with Alzheimer's disease and aging. Neurosci. Lett. 119, 182-186

7. Hof P. R., Delacourte A. and Bouras C. (1992) Distribution of cortical neurofibrillary tangles in progressive supranuclear palsy: a quantitative analysis of six cases. Acta Neuropathol. 84, 45-51

8. Horoupian D. S. and ChU P. L. (1994) Unusual case of corticobasal degeneration with tau/Gallyas-positive neuronal and glial tangles. Acta Neuropathol. 88, 592-598

9. Hyman B. T., Van Hoesen G. W., Wolozin B. L., Davies P., Kromer L. J. and Damasio A. R. (1988) Alz-50 antibody recognizes Alzheimer-related neuronal changes. Annu. Neurol. 23, 371-379

10. Iqbal K., Alonso A., Gong C., Khatoon S., Kudo T., SingH T. and GrundKE-IQBAL I. (1993) Molecular pathology of Alzheimer neurofibrillary degeneration. Acta Neurobiol. Exp. 53, 325-335

11. Iwatsubo T., Hasegawa M. and Ihara Y. (1994) Neuronal and glial tau-positive inclusions in diverse neurologic diseases share common phosphorylation characteristics. Acta Neuropathol. 88, 129-136

12. Joachim C. L., Morris J. H., Selkoe D. J. and Kosik K. S (1987) Tau epitopes are incorporated into a range of lesions in Alzheimer's disease. J. Neuropathol. Exp. Neurol. 46, $611-622$

13. KidA E., BarciKowsKa M. and NiemCzewsKa M. (1992) Immunohistochemical study of a case with progressive supranuclear palsy without ophthalmoplegia. Acta Neuropathol. 83, 328-332

14. Kimura T., Ono T., Takamatsu J., Yamamoto H., IkegaMI, K., Kondo A., Hasegawa M., Ihara Y., Miyamoto E. and MiYakawa T. (1996) Sequential changes of tau-sitespecific phosphorylation during development of paired helical filaments. Dementia 7, 177-181

15. Li F., Iseki E., Kosaka K., Nishimura T., Akiyama H. and Kato M. (1996) Progressive supranuclear palsy with frontotemporal atrophy and various tau-positive abnormal structures. Clin. Neuropathol. 15, 319-323
16. Matsumoto S., Udaka F., Kameyama M., Kusaka H., ITo H. and IMAI T. (1996) Subcortical neurofibrillary tangles, neuropil threads, and argentophilic glial inclusions in corticobasal degeneration. Clin. Neuropathol. 15, 209-214

17. Mori H., Nishimura M., Namba Y. and OdA M. (1994) Corticobasal degeneration: a disease with widespread appearance of abnormal tau and neurofibrillary tangles, and its relation to progressive supranuclear palsy. Acta Neuropathol. 88, 113-121

18. Papasozomenos S. C. (1989) Tau protein immunoreactivity in dementia of the Alzheimer type. I. Morphology, evolution, distribution, and pathogenetic implications. Lab. Invest. 60, 123-137

19. Papasozomenos S. C. (1989) Tau protein immunoreactivity in dementia of the Alzheimer type. II. Electron microscopy and pathogenetic implications. Effects of fixation on the morphology of Alzheimer's abnormal filaments. Lab. Invest. 60, 375-389

20. Paulus W. and Selim M. (1990) Corticonigral degeneration with neuronal achromasia and basal neurofibrillary tangles. Acta Neuropathol. 81, 89-94

21. Schmit M. L., Huang R., Martin J. A., Henley J., Mawal-Denan M., Hurtig H., Lee V. M. Y. and TroJANOWSKI J. Q. (1996) Neurofibrillary tangles in progressive supranuclear palsy contain the same tau epitopes identified in Alzheimer's disease PHF tau. J. Neuropathol. Exp. Neurol. 55, 534-539

22. Shin R. W., Kitamoto T. and Tateishi J. (1991) Modified tau is present in younger nondemented persons: a study of subcortical nuclei in Alzheimer's disease and progressive supranuclear palsy. Acta Neuropathol. 81, 517-523

23. Tabaton M., Whitehouse P. J., Perry G., Davies P., Autilio-Gambetti L. and Gambetti P. (1988) Alz 50 recognizes abnormal filaments in Alzheimer's disease and progressive supranuclear palsy. Annu. Neurol. 24, 407-413

24. TomonaGa M. (1977) Ultrastructure of neurofibrillary tangles in progressive supranuclear palsy. Acta Neuropathol. 37, 177-181

25. Uchihara T., Mitami K., Mori H., Yamada M. and Ikeda K. (1994) Abnormal cytoskeletal pathology peculiar to corticobasal degeneration is different from that of Alzheimer's disease or progressive supranuclear palsy. Acta Neuropathol. 88, 379-383

26. Wakabayashi K., Oyanagi K., Makifuchi T., IkUta F., Homma A., Homma Y., Horikawa Y. and Tokiguchi S. (1994) Corticobasal degeneration: etiopathological significance of the cytoskeletal alteration. Acta Neuropathol. 87, $545-553$

27. Wolozin B. L., Pruchnicki A., Dickson D. W. and Devies P. (1986) A neuronal antigen in the brains of Alzheimer patients. Science 232, 648-650

28. Yagishita S., Itoh Y., Amano N., Nakano T. and Saitoh A. (1979) Ultrastructure of neurofibrillary tangles in progressive supranuclear palsy. Acta Neuropathol. 48, 27-30 